\title{
Resolución 73/257 de la Asamblea General de la ONU sobre el cumplimiento inmediato del fallo Avena
}

Pablo Arrocha Olabuenaga*

El 14 de noviembre de 2018 fue ejecutado en Texas el señor. Roberto Ramos Moreno, convirtiéndose en el sexto mexicano ejecutado en absoluta violación al fallo emitido por la Corte Internacional de Justicia (CIJ) en el caso Avena, el 31 de marzo de 2004. ${ }^{1}$ Este Anuario ha publicado en el pasado artículos dedicados tanto a este caso $^{2}$ como al primero de incumplimiento ante la ejecución de José Ernesto Medellín Rojas, ${ }^{3}$ que implicó una vuelta a la CIJ mediante el recurso de interpretación del fallo en 2008. Quince años después de su emisión, la sentencia de la Corte no sólo sigue sin cumplirse; las ejecuciones de José Ernesto Medellín Rojas (2008), Humberto Leal García (2011), Edgar Tamayo Arias (2014), Ramiro Hernández Llanas (2014), Rubén Cárdenas Ramírez (2017) y Roberto Ramos Moreno

* Asesor jurídico de la Misión Permanente de México ante la ONU.

1 Véase Secretaría de Relaciones Exteriores, "México condena la ejecución en Texas del connacional Roberto Ramos Moreno", comunicado núm. 308. Disponible en: https:// www.gob. $\mathrm{mx} /$ sre/prensa / mexico-condena-la-ejecucion-en-texas-del-connacional-roberto-ramosmoreno?state $=$ published.

2 Gómez Robledo, Juan Manuel, "El caso Avena y otros nacionales mexicanos (México c. Estados Unidos de América) ante la Corte Internacional de Justicia”, Anuario Mexicano de Derecho Internacional, México, vol. V, 2005, pp. 173-220.

3 Arrocha Olabuenaga, Pablo, "Caso Medellín vs. Texas. Comentarios al fallo de la Suprema Corte de los Estados Unidos, así como, a la solicitud de interpretación del fallo Avena del 31 de marzo de 2004, presentada a la Corte Internacional de Justicia por México en junio de 2008”, Anuario Mexicano de Derecho Internacional, vol. IX, 2009, pp. 677-705. 
(2018), han constituido nuevas violaciones por parte de Estados Unidos de América (EUA) a sus obligaciones internacionales, generando daños adicionales a México.

De conformidad con el artículo 94 (2) de la Carta de la ONU, "si una de las partes en un litigio dejare de cumplir las obligaciones que le imponga un fallo de la Corte, la otra parte podrá recurrir al Consejo de Seguridad, el cual podrá, si lo cree necesario, hacer recomendaciones o dictar medidas con el objeto de que se lleve a efecto la ejecución del fallo". ${ }^{4}$ México ha enviado tres cartas al Consejo de Seguridad, en 2014, 2017 y 2018 respectivamente, informando y condenando estas violaciones. A la fecha, ninguna de ellas ha recibido respuesta.

En ese contexto, habiendo agotado las vías judiciales, y tras la imposibilidad de contar con el respaldo del Consejo de Seguridad tomando en cuenta la calidad de EUA como miembro permanente de dicho órgano, México decidió presentar un proyecto de resolución ante la Asamblea General de la ONU (AGONU) pidiendo urgentemente el cumplimiento pleno e inmediato del fallo de la Corte Internacional de Justicia de 31 de marzo de 2004, al amparo del artículo 10 de la Carta de la ONU, el cual establece que

La Asamblea General podrá discutir cualesquier asuntos o cuestiones dentro de los límites de esta Carta o que se refieran a los poderes y funciones de cualquiera de los órganos creados por esta Carta, y salvo lo dispuesto en el Artículo 12 podrá hacer recomendaciones sobre tales asuntos o cuestiones a los Miembros de las Naciones Unidas o al Consejo de Seguridad o a éste y a aquéllos. ${ }^{5}$

El proyecto "Fallo de la Corte Internacional de Justicia del 31 de marzo de 2004 en la causa relativa a Avena y otros nacionales mexicanos: necesidad de cumplimiento inmediato", registrado bajo el tema de agenda "Fortalecimiento del Sistema de Naciones Unidas", fue adoptado por la AGONU el 20 de diciembre de 2018 como la resolución 73/257, con 69 votos a favor, 4 en contra y 66 abstenciones.

Cabe destacar que, esta resolución constituye el segundo precedente de la AGONU llamando al cumplimiento de un fallo de la CIJ, el primero siendo la resolución 41/31, intitulada "Sentencia de la Corte Internacional

4 Disponible en: https://www.un.org/es/sections/un-charter/chapter-xiv/index.html.

5 Disponible en: https://www.un.org/es/sections/un-charter/chapter-iv/index.html. 
de Justicia del 27 de junio de 1986 sobre las actividades militares y paramilitares en y contra Nicaragua: necesidad de su inmediato cumplimiento". ${ }^{6}$ Ambos precedentes tienen como común denominador el incumplimiento de una sentencia por parte de EUA.

La resolución 73/257 se compone de seis párrafos preambulares y un sólo párrafo operativo, en el que la AGONU "pide urgentemente que se cumpla plena e inmediatamente el fallo de la Corte Internacional de Justicia del 31 de marzo de 2004”. En el preámbulo se hace referencia a la resolución 41/31, se reconoce a la CIJ como el principal órgano judicial de la ONU y se refrenda la obligatoriedad de sus fallos, y se hace referencia a las dos sentencias de la CIJ relativas al caso Avena. El elemento más novedoso del texto es que reconoce de manera explícita que las seis ejecuciones llevadas a cabo hasta la fecha en contravención del fallo “constituye(n) un nuevo incumplimiento de las obligaciones internacionales que incumben a los Estados Unidos de América y causa(n) un daño adicional a México".

Como era de esperarse, hubo mucha resistencia por parte de algunas delegaciones respecto del proyecto de resolución. Hay que tomar en cuenta que menos de la mitad de la membresía de la ONU reconoce la jurisdicción obligatoria de la CIJ. Además, para muchos se trataba de un asunto bilateral que no debía ventilarse en la AGONU, y que sentaría un precedente "peligroso", al ser la AGONU quien se pronunciase sobre casos de incumplimiento de sentencias. Por su parte, la entonces Representante Permanente de EUA ante la ONU, envió una nota a todas las delegaciones en Nueva York, solicitando oponerse a esta iniciativa, lo cual elevó el nivel de tensión en torno a esta resolución.

El gobierno de México siempre ha reconocido y apreciado los esfuerzos del Departamento de Estado y otras autoridades de EUA en torno a la implementación del fallo. ${ }^{7}$ No obstante, México sostiene que el incumplimiento de las sentencias de la CIJ no es una preocupación de orden estrictamente bilateral, sino que quebranta el Estado de derecho internacional e impacta

6 Disponible en: https: / / undocs.org/es/A/RES/41/31.

7 Véase Secretaría de Relaciones Exteriores, “AGONU aprueba la resolución «Fallo de la Corte Internacional de Justicia del 31 de marzo de 2004 sobre Avena y otros ciudadanos mexicanos»", comunicado núm. 017. Disponible en: https://www.gob. mx/sre/prensa/la-asamblea-general-de-naciones-unidas-aprobo-la-resolucion-fallo-de-la-corte-internacional-de-justicia-del31-de-marzo-de-2004-sobre-avena-y-otros-ciudadanos-mexicanos. 
profundamente al sistema de las Naciones Unidas en su conjunto. Por esos motivos, el texto de la resolución es estrictamente jurídico y técnico.

En suma, el proceso hacia la adopción de la Resolución 73/257 no fue fácil, y requirió de una intensa labor diplomática tanto en la sede de la ONU, a cargo de la Misión Permanente de México ante la ONU, como en la propia cancillería, especialmente por parte de la Consultoría Jurídica, así como, del apoyo de otras representaciones de México en el exterior. Es importante tomar en cuenta que el proyecto de resolución fue registrado ante la Secretaría de la ONU el 10 de diciembre de 2018, es decir, tan sólo diez días antes de su consideración y adopción en sesión plenaria de la AGONU. Lograr la adopción de una resolución de esta naturaleza a la luz del ambiente parlamentario en la ONU y contando con tan poco tiempo para su cabildeo representa una gran victoria para México en el ámbito multilateral.

En conferencia de prensa, tras la adopción de la Resolución 73/257, el canciller mexicano, Marcelo Ebrard, declaró:

El día de ayer se obtuvo una votación histórica en las Naciones Unidas en materia de protección de los derechos humanos a nuestros nacionales en los Estados Unidos por el caso Avena... esto sienta un precedente de la mayor magnitud para la protección de nuestros connacionales en los Estados Unidos. ${ }^{8}$

La Resolución 73/257, en efecto, representa un precedente histórico para la causa de México en protección de sus connacionales en el extranjero. Con esta iniciativa, México refrendó su respeto por el Estado de derecho y el derecho internacional. Más aún, al adoptar la resolución, la propia AGONU hizo suya la condena a la falta de rendición de cuentas, apostando por el fortalecimiento tanto de la CIJ como de las Naciones Unidas en su conjunto, y de la solución pacífica de controversias, elementos fundamentales para la plena realización de los principios y propósitos de la Organización.

8 Disponible en: https://www.excelsior.com. $m x /$ nacional/reitera-ebrard-beneplacito-por-fallode-onu-sobre-caso-avena/1286355. 
Esta revista forma parte del acervo de la Biblioteca Jurídica Virtual del Instituto de Investigaciones Jurídicas de la UNAM

\section{Resolución aprobada por la Asamblea General el 20 de diciembre de 2018}

[sin remisión previa a una Comisión Principal (A/73/L.63)]

73/257. Fallo de la Corte Internacional de Justicia de 31 de marzo de 2004 en la causa relativa a Avena y otros nacionales mexicanos: necesidad de cumplimiento inmediato

La Asamblea General,

Recordando su resolución 41/31, de 3 de noviembre de 1986,

Observando que, de conformidad con la Carta de las Naciones Unidas, la Corte Internacional de Justicia es el principal órgano judicial de las Naciones Unidas y que cada Miembro se compromete a cumplir la decisión de la Corte en todo litigio en que sea parte,

Consciente del fallo de la Corte Internacional de Justicia de 31 de marzo de 2004 en la causa relativa a Avena y otros nacionales mexicanos ${ }^{1}$, referente a la violación de la obligación de proporcionar información consular sin demora y permitir a los funcionarios consulares comunicarse con los nacionales de su Estado, tener acceso a ellos, visitarlos y organizar su defensa ante los tribunales, de conformidad con la Convención de Viena sobre Relaciones Consulares, de 24 de abril de $1963^{2}$, respecto de 52 nacionales mexicanos que habían sido condenados a muerte en ciertos estados de los Estados Unidos de América, y en particular del párrafo 153 9) de dicho fallo, en el que se estableció que la reparación adecuada en dicha causa consistía en la obligación de los Estados Unidos de América de proceder, por medios de su propia elección, al examen y la reconsideración de las declaraciones de culpabilidad y las condenas de 51 nacionales mexicanos mencionados en el fallo,

Consciente también del fallo de la Corte Internacional de Justicia de 19 de enero de 2009 , relativo a la solicitud de interpretación del fallo de 31 de marzo de $2004^{3}$,

${ }^{1}$ Documentos Oficiales de la Asamblea General, quincuagésimo noveno periodo de sesiones, Suplemento núm. 4 (A/59/4), cap. V, secc. A.23.

${ }^{2}$ Naciones Unidas, Treaty Series, vol. 596, núm. 8638.

${ }^{3}$ Documentos Oficiales de la Asamblea General, sexagésimo cuarto periodo de sesiones, Suplemento núm. 4 (A/64/4), cap. V, secc. B.12. 
en particular de su párrafo 61 2), en el que se estableció que, en el caso del Sr. José Ernesto Medellín Rojas, los Estados Unidos de América habían incumplido la obligación que les incumbía en virtud de la providencia sobre medidas provisionales de 16 de julio de $2008^{4}$,

Habiendo considerado que, hasta la fecha, no se han llevado a cabo respecto de los nacionales mexicanos mencionados en el fallo de 31 de marzo de 2004 ni la revisión ni la reconsideración impuestas en él,

Considerando que seis de los nacionales mexicanos mencionados en el fallo de 31 de marzo de 2004 han sido ejecutados ${ }^{5}$, en clara violación de la decisión de la Corte, lo cual constituye un nuevo incumplimiento de las obligaciones internacionales que incumben a los Estados Unidos de América y causa un daño adicional a México,

Pide urgentemente que se cumpla plena e inmediatamente el fallo de la Corte Internacional de Justicia de 31 de marzo de 2004 en la causa relativa a Avena y otros nacionales mexicanos ${ }^{1}$, de conformidad con las disposiciones pertinentes de la Carta de las Naciones Unidas.

$63^{a}$ sesión plenaria

20 de diciembre de 2018

${ }^{4}$ Ibid., sexagésimo tercer periodo de sesiones, Suplemento núm. 4 (A/63/4), cap. V, secc. B.15.

5 José Ernesto Medellín Rojas (2008), Humberto Leal García (2011), Edgar Tamayo Arias (2014), Ramiro Hernández Llanas (2014), Rubén Cárdenas Ramírez (2017) y Roberto Ramos Moreno (2018). 
Esta revista forma parte del acervo de la Biblioteca Jurídica Virtual del Instituto de Investigaciones Jurídicas de la UNAM https://www.juridicas.unam.mx/ https://biblio.juridicas.unam.mx/bjv

https://revistas.juridicas.unam.mx/

DOI: http://dx.doi.org/10.22201/iij_24487872e_2020.20.14485

2. Patrón de votación de la resolución $73 / 257$

General Assembly

General Assembly: 63rd Plenary meeting

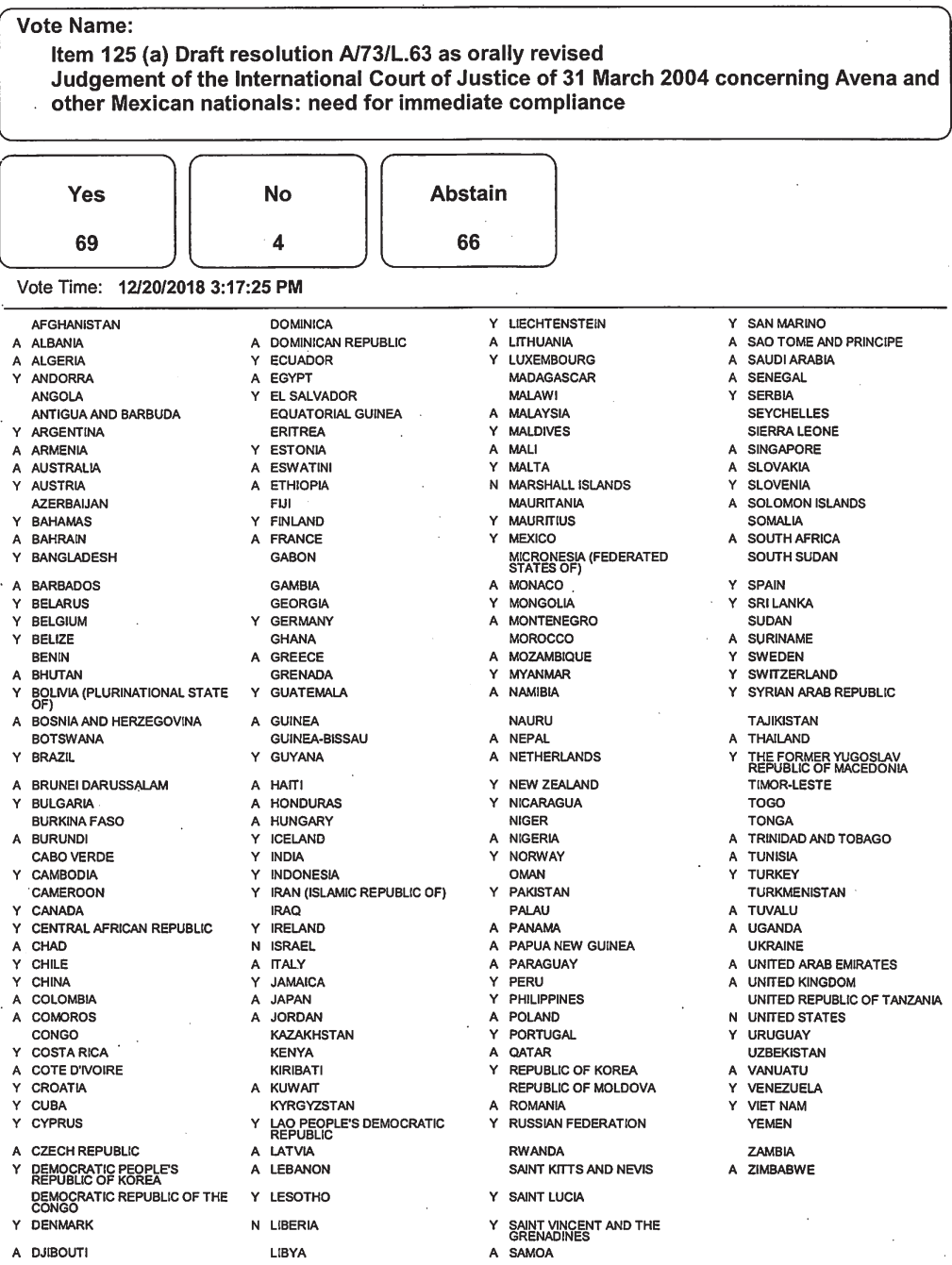


Esta revista forma parte del acervo de la Biblioteca Jurídica Virtual del Instituto de Investigaciones Jurídicas de la UNAM https://www.juridicas.unam.mx/

DOI: http://dx.doil.org/10.22201/iij.24487872e_2020.20.14485

3. Intervención del Representante Permanente de México ante la $O N U$ introduciendo el proyecto de resolución ante la $A G O N U$

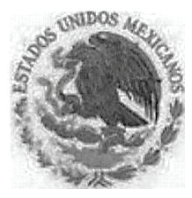

Misión PeRmanente de MÉXICO

\section{Introducción del proyecto de resolución L.63: \\ "Fallo de la Corte Internacional de Justicia de 31 de marzo de 2004 en la causa relativa a Avena y otros nacionales mexicanos: necesidad de cumplimiento inmediato"}

\section{$73^{\circ}$ Asamblea General de las Naciones Unidas}

(Nueva York, 20 de diciembre de 2018)

Señora presidenta:

A nombre del Gobierno de México, tengo el honor de introducir el proyecto de resolución contenido en el documento L.63, intitulado "Fallo de la Corte Internacional de Justicia de 31 de marzo de 2004 en la causa relativa a Avena y otros nacionales mexicanos: necesidad de cumplimiento inmediato".

El 31 de marzo de 2004 la Corte Internacional de Justicia emitió su sentencia en un caso presentado por México en relación con la violación a la Convención de Viena sobre Relaciones Consulares de 1963 y al debido proceso respecto de más de 50 casos de mexicanos condenados a pena de muerte en los Estados Unidos.

La Corte determinó que, en efecto, los Estados Unidos habían violado su obligación de proveer la información consular requerida, así como de permitir a México ejercer su derecho a la asistencia consular, de conformidad con la Convención de 1963. En ese sentido, la Corte ordenó la revisión y reconsideración de 51 casos de mexicanos mencionados en el fallo.

Casi 15 años han pasado desde la emisión de la sentencia y, a la fecha, ésta sigue sin cumplirse. En respuesta a este estado de incumplimiento, México recurrió nuevamente a la Corte Internacional de Justicia en 2008 y ha enviado tres cartas al Consejo de Seguridad haciendo de su conocimiento estas violaciones en 2014, 2017 y 2018 . Hasta ahora, México no ha recibido respuesta a estas cartas por parte del Consejo de Seguridad. 
Esta revista forma parte del acervo de la Biblioteca Jurídica Virtual del Instituto de Investigaciones Jurídicas de la UNAM

A lo largo de estos 14 años, México ha buscado la implementación del fallo en conjunto con el Departamento de Estado y otras autoridades de los Estados Unidos.

A pesar de estos esfuerzos, que el Gobierno mexicano reconoce y aprecia, seis ciudadanos mexicanos han sido ejecutados en el estado de Texas, sin respetar la orden de la Corte para revisar y reconsiderar sus veredictos de culpabilidad y sentencias. La última ejecución tuvo lugar hace apenas un mes. Dichos actos causan violaciones adicionales al Estado Mexicano.

En estas circunstancias, agotados ya todos estos esfuerzos, y de conformidad con el Artículo 10 de la Carta de las Naciones Unidas, el Gobierno de México ha decidido recurrir a la Asamblea General. El incumplimiento de las sentencias de la Corte Internacional de Justicia, que es el principal órgano judicial de esta Organización, no es una preocupación de orden estrictamente bilateral, sino que quebranta el estado de derecho internacional e impacta profundamente al sistema de las Naciones Unidas en su conjunto.

Por estos motivos, México ha registrado este proyecto de resolución bajo el tema 125(a) de la agenda de la Asamblea General sobre el "Fortalecimiento del sistema de Naciones Unidas". El texto de la resolución es jurídico y técnico. Para simplificar aún más el texto y evitar consideraciones de carácter político, quiero presentar formalmente una enmienda al documento L.63 consistente en la eliminación del segundo párrafo operativo. Solicitamos que la Asamblea General tome acción sobre el texto tal y como ha sido enmendado oralmente.

Finalmente, reitero la voluntad del Gobierno de México para seguir colaborando con las autoridades de los Estados Unidos en la implementación del Fallo Avena.

Gracias. 
Esta revista forma parte del acervo de la Biblioteca Jurídica Virtual del Instituto de Investigaciones Jurídicas de la UNAM

\title{
4. Nota de la Representante Permanente de EUA ante la ONU dirigida a todas las delegaciones
}

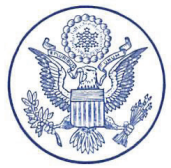

\author{
THE REPRESENTATIVE \\ OF THE \\ UNITED STATES OF AMERICA \\ TO THE \\ UNITED NATIONS
}

December 20, 2018

Excellency,

I write to you regarding the General Assembly resolution proposed by Mexico on the Avena case. The General Assembly is scheduled to take action on this resolution today, December 20. The United States believes that it is inappropriate that Mexico has brought this bilateral matter to the UN General Assembly. We are also disappointed Mexico failed to consult with the United States prior to circulating the draft resolution. We will vote "no" on this resolution. Our vote should not be interpreted as a repudiation of our international obligations regarding consular notification and access. On the contrary, the United States continues to take very seriously our international obligations with respect to consular notification and access. We will vote "no" to affirm that the UN General Assembly is not the appropriate venue for this issue.

The United States continues to take steps with respect to the Avena judgment, and we have engaged in close and extensive consultations with Mexico. The United States notes that the United States Supreme Court has held, in Medellin v. Texas, that the ICJ's Avena decision does not constitute directly enforceable federal law and that U.S. obligations could be discharged through the adoption of federal legislation. This resolution will not alter the force of the Supreme Court's decision as binding upon the United States government. Accordingly, legislation that would facilitate actions consistent with the Avena judgment in the United States was included in the President's Fiscal Year 2019 budget request.

The United States has closely consulted with Mexico on its efforts to implement the Avena judgment, and has kept Mexico informed of its efforts. Mexico's decision to introduce this resolution was unfortunate. We call on all delegations to vote "no" on this resolution.

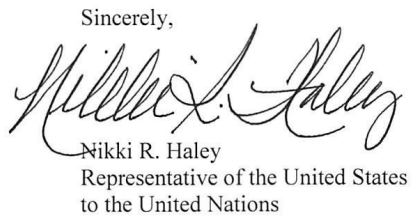

Permanent Representatives to the United Nations

New York, NY 
Esta revista forma parte del acervo de la Biblioteca Jurídica Virtual del Instituto de Investigaciones Jurídicas de la UNAM https://www.juridicas.unam.mx/

\title{
5. Cartas remitidas por los secretarios de Relaciones Exteriores de México, José Antonio Meade y Luis Videgaray, respectivamente, al Consejo de Seguridad en 2014, 2017 y 2018
}

A. José Antonio Meade Kuribreña (2014)

\author{
Embajadora Sylvie Lucas \\ Presidenta del Consejo de Seguridad de las Naciones Unidas \\ Representante Permanente de Luxemburgo \\ ante la Organización de las Naciones Unidas \\ Nueva York
}

Señora Presidenta:

Tengo el honor de dirigirme a Usted en relación con la falta de cumplimiento por parte del Gobierno de Estados Unidos de América de la sentencia de la Corte Internacional de Justicia (ClJ) dictada el 31 de marzo de 2004 en el Caso concerniente a Avena y otros nacionales mexicanos (México c. Estados Unidos).

Dicho fallo resolvió una controversia relacionada con el proceso penal de 51 mexicanos condenados a pena de muerte, cuyo derecho a la notificación y asistencia consular prevista en el artículo 36 de la Convención de Viena sobre Relaciones Consulares (CVRC) no fue respetado, contraviniendo su derecho al debido proceso judicial. En el resolutivo (9) de la sentencia, la CIJ impuso a Estados Unidos la obligación de proporcionar, a través de los medios de su propia elección, la revisión y reconsideración de los veredictos de culpabilidad y de las sentencias de los mexicanos contenidos en el fallo.

Posteriormente, en 2008, ante la inminente ejecución del mexicano José Ernesto Medellín Rojas, México presentó ante la $\mathrm{CIJ}$ una solicitud de interpretación sobre el sentido y alcance del Fallo Avena de 2004. Consecuentemente, en su Ordenanza del 16 de julio de 2008, la Corte ordenó a Estados Unidos que adoptara "todas las medidas necesarias" para asegurar que los nacionales mexicanos incluidos en dicha petición no fuesen ejecutados, a menos que y hasta que sus casos hubiesen sido revisados y reconsiderados. Asimismo, en el subsecuente fallo de la ClJ del 19 de enero de 2009, se estableció que Estados Unidos violó sus obligaciones internacionales vinculantes mediante la ejecución de José Ernesto Medellín Rojas. 
Hasta la fecha, no sólo Estados Unidos ha incumplido con las obligaciones impuestas por la ClJ sino que también tres de los 51 mexicanos incluidos en el Fallo Avena han sido ejecutados en el estado de Texas sin que sus casos hayan sido revisados ni reconsiderados, en desafío a la Corte: José Ernesto Medellín Rojas (2008), Humberto Leal García (2011) y, recientemente, Edgar Tamayo Arias, el 22 de enero de 2014. Estos actos han causado un daño adicional a los derechos de México al amparo de la citada sentencia. Traigo asimismo a su atención que se ha fijado fecha de ejecución en el mes de abril para un cuarto mexicano incluido en el fallo, el señor Ramiro Hernández Llanas.

El Gobierno de Estados Unidos ha subrayado la importancia de que dicho país cumpla con las obligaciones impuestas por el fallo. El Gobierno de México reconoce los amplios esfuerzos que, en su momento, realizó para ello el Presidente George W. Bush, así como los que lleva a cabo la actual administración del Presidente Barack Obama, y en particular el Secretario de Estado John Kerry, y reconoce asimismo las acciones de algunos miembros del Congreso de Estados Unidos. Sin embargo, a diez años de que la ClJ emitiera su decisión, el cumplimiento de ésta continúa pendiente.

En consideración de lo anterior, el Gobierno de México, indignado por la ejecución de ciudadanos mexicanos en contravención a lo ordenado por la $\mathrm{ClJ}$, y preocupado por el efecto negativo de esta situación, en el régimen internacional de asistencia y protección consular, desea hacer del conocimiento del Consejo de Seguridad la apremiante y grave situación que dicho incumplimiento representa, y se reserva el derecho que le asiste conforme al párrafo 2 del Artículo 94 de la Carta de las Naciones Unidas.

Aprovecho esta oportunidad para reiterar a usted las seguridades de mi más atenta y distinguida consideración.

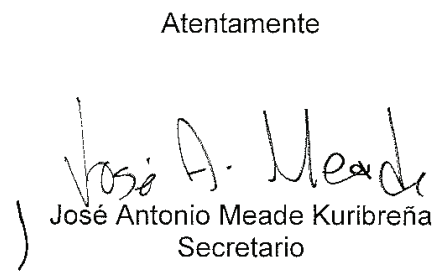


Esta revista forma parte del acervo de la Biblioteca Jurídica Virtual del Instituto de Investigaciones Jurídicas de la UNAM https://www.juridicas.unam.mx/

B. Luis Videgaray Caso (2017)

\section{Ambassador Sebastiano Cardi \\ President of the United Nations Security Council \\ Permanent Representative of Italy to the United Nations \\ New York}

\section{Mr. President,}

I have the honor to address Your Excellency regarding the United States Government's lack of compliance with the judgment that the International Court of Justice (ICJ) rendered on March 31, 2004, in the Case Concerning Avena and other Mexican nationals (Mexico v. United States).

The judgment settled a controversy with respect to the judicial processes of 51 Mexican nationals sentenced to death, whose right to consular notification and assistance in accordance with article 36 of the Vienna Convention on Consular Relations (VCCR) was not respected, hence contravening their right to a fair trial. In the operative paragraphs of its decision, the ICJ imposed an obligation upon the United States to review and reconsider, by means of its own choosing, the convictions and sentences of the Mexican nationals listed in the judgment.

Moreover, in 2008, faced with the imminent execution of José Ernesto Medellín Rojas, Mexico requested an interpretation of the means and scope of the 2004 Avena judgment before the $\mid \mathrm{CJ}$, together with a request for the indication of provisional measures. In its Order of July 16, 2008 on the provisional measures requested by Mexico, the Court ordered the United States to adopt "all necessary measures" to guarantee that the Mexican nationals listed in the request were not executed unless and until their cases were reviewed and reconsidered. Subsequently, in its judgment of January 19,2009, the ICJ determined that by executing José Ernesto Medelín Rojas, the United States had breached its legally binding international obligations. 
Esta revista forma parte del acervo de la Biblioteca Jurídica Virtual del Instituto de Investigaciones Jurídicas de la UNAM https://www.juridicas.unam.mx/

https://biblio.juridicas.unam.mx/bjv

https://revistas.juridicas.unam.mx/

DOI: http://dx.doi.org/10.22201/iij_24487872e_2020.20.14485

To this date, the United States has not complied with the obligations arising from the judgment of the ICJ, and in fact, four of the 51 Mexicans identified in the Avena judgment have been executed in Texas without their cases being reviewed nor reconsidered, in defiance to the Court's authority: José Ernesto Medellín Rojas (2008), Humberto Leal García (2011), Edgar Tamayo Arias (2014), and Ramiro Hernández Llamas (2014). These acts have caused additional damages to Mexico's rights under the Avena judgment. I would like to take this opportunity to bring to your attention the fact that another date of execution has been set in the upcoming month of November for a fifth Mexican national included in the judgment, Mr. Rubén Cárdenas Ramírez.

Taking these elements into consideration, outraged by the execution of four Mexican nationals and the announcement of a fifth execution in contravention of the ICJ's decisions, and concerned with the negative effect that this situation causes to the international regime of consular protection and assistance, the Mexican Government wishes to inform the Security Council of the grave and urgent situation that this case of non-compliance represents, and it reserves its rights in accordance with article 94, paragraph 2, of the United Nations Charter.

1 avail myself this opportunity to reiterate the assurances of my most distinguished consideration.

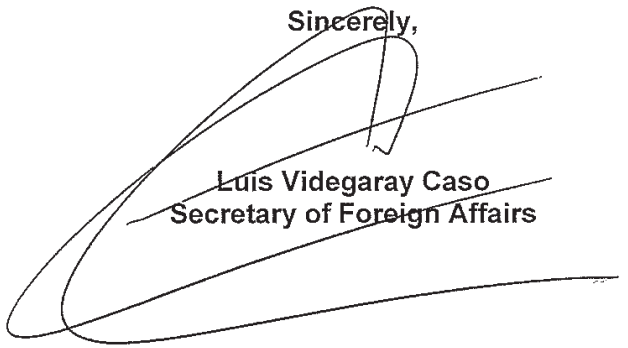


Esta revista forma parte del acervo de la Biblioteca Jurídica Virtual del Instituto de Investigaciones Jurídicas de la UNAM https://www.juridicas.unam.mx/ https://biblio.juridicas.unam.mx/bjv

https://revistas.juridicas.unam.mx/

DOI: http://dx.doi.org/10.22201/iij_.24487872e_2020.20.14485

C. Luis Videgaray Caso (2018)

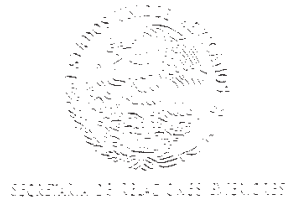

Mexico City

October $24^{\text {th }}, 2018$

\author{
Ambassador Sacha Sergio Llorenti Solíz \\ President of the United Nations Security Council \\ Permanent Representative of Bolivia to the United Nations \\ New York
}

\title{
Mr. President,
}

1 have the honor to address Your Excellency regarding the United States Government's persistent lack of compliance with the judgment that the International Court of Justice (ICJ) rendered on March 31, 2004, in the Case Concerning Avena and Other Mexican Nationals (Mexico v. United States of America).

The judgment settled a controversy concerning the judicial processes afforded to 51 Mexican nationals sentenced to death in the United States whose right to consular notification and assistance, in accordance with article 36 of the Vienna Convention on Consular Relations (VCCR), was violated, contravening their right to a fair trial in their respective capital prosecutions. In the operative paragraphs of its decision, the ICJ imposed an obligation upon the United States to review and reconsider, by means of its own choosing, the convictions and sentences of the Mexican nationals listed in the judgment.

Several years later, faced with the imminent execution of Mr. José Ernesto Medellín Rojas in 2008, Mexico requested an interpretation of the means and scope of the 2004 Avena judgment from the ICJ, together with a request for provisional measures to halt the executions of the individuals at greatest risk. In its Order on the requested provisional measures, issued on July 16,2008, the Court ordered the United States to adopt "all necessary measures" to guarantee that Mr. Medellin and the four other Mexican nationals listed in the request were not executed unless and until their cases were reviewed and reconsidered. Nevertheless, the United States executed Mr. Medellín. Subsequently, in its judgment of January 19,2009 , the ICJ determined that by executing Mr. Medellin, the United States had breached its legally binding international obligations.

To this day, the United States has not complied with the obligations arising from the judgment of the $\mathrm{ICJ}$, and in fact, five of the 51 Mexicans identified in the Avena judgment have been executed in Texas without the required review and reconsideration, in defiance of the Court's authority: José Ernesto Medellín Rojas (2008), Humberto Leal García (2011), Edgar Tamayo Arias (2014), Ramiro Hernández Llamas (2014), and Rubén Cárdenas Ramírez (2017). These acts have caused additional damages to Mexico's rights under the Avena judgment. 
Esta revista forma parte del acervo de la Biblioteca Jurídica Virtual del Instituto de Investigaciones Jurídicas de la UNAM https://www.juridicas.unam.mx/ https://biblio.juridicas.unam.mx/bjv

https://revistas.juridicas.unam.mx/

DOI: http://dx.doi.org/10.22201/iij.24487872e_2020.20.14485

I would like to take this opportunity to bring to your attention the fact that another date of execution has been set in the upcoming month of November for a sixth Mexican national included in the judgment, Mr. Roberto Ramos Moreno. ${ }^{1}$ Three of the five Mexican nationals specifically named in the July 16, 2008 provisional measures have now been executed in defiance of that order; Mr. Ramos Moreno would be the fourth.

It is important to mention that Mr. Ramos Moreno was provided an incompetent attorney who did not undertake any of the investigation necessary for the sentencing phase of a death penalty case and consequently presented no evidence at all in Mr. Ramos Moreno's defense at his sentencing hearing. Additionally, Texas authorities failed to inform Mr. Ramos Moreno of his right to seek consular assistance as established in the Vienna Convention on Consular Relations (VCCR); as a direct result, Mexico was prevented from providing critical assistance during the entire period between his arrest and the start of his trial nearly eleven months later. It was during this period that Mexico would have been best able to assist with the investigation and ensure that Mr. Ramos Moreno received an adequate defense.

Taking these elements into consideration, outraged by the execution of five Mexican nationals and the announcement of a sixth execution in contravention of the ICJ's decisions, and concerned with the negative impact that this situation has on the international regime of consular protection and assistance, the Mexican Government wishes to inform the Security Council of the grave and urgent situation that this case of non-compliance represents, and it reserves its rights in accordance with article 94 , paragraph 2, of the United Nations Charter.

I avail myself of this opportunity to reiterate the assurances of my most distinguished consideration.

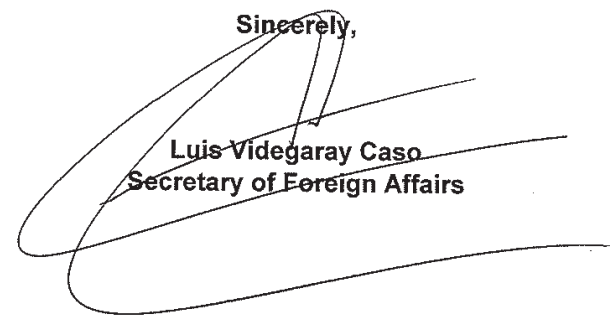

"In the documents for the Avena case, this individual was listed as Roberto Moreno Ramos, and is referred to in many U.S.
court documents as Robert Moreno Ramos. However, the name on his Mexican birth certificate is Roberto Ramos Moreno. 